\title{
Diagnosis-related deterioration of lung function after extracorporeal membrane oxygenation
}

\author{
Marjolein Spoel*,+, Roxanne Laas*,+, Saskia J. Gischler*, Wim J.C. Hop\#, \\ Dick Tibboel*, Johan C. de Jongste and Hanneke ljsselstijn*
}

ABSTRACT: The aim of the study was to assess lung function longitudinally after neonatal extracorporeal membrane oxygenation (ECMO), and to identify any effects of diagnosis and perinatal characteristics.

121 neonatal ECMO-treated children (70 with meconium aspiration syndrome, 20 congenital diaphragmatic hernia and 31 with other diagnoses) performed a total of 191 lung function measurements at 5, 8 and/or 12 yrs. We assessed dynamic and static lung volumes, reversibility of airway obstruction and diffusion capacity.

Mean SDS forced expiratory volume in $1 \mathrm{~s}$ (FEV1) at 5 yrs before and after bronchodilation $(-0.51$ and 0.07$)$ was significantly higher than at $8(-0.79$ and $-0.4 ; p<0.04)$ and 12 yrs $(-1.10$ and -0.52; $p<0.003)$. Mean SDS for all spirometric parameters before and after bronchodilation were significantly lower in the congenital diaphragmatic hernia group compared with the other diagnostic groups (all $p \leqslant 0.025$ ). A significant volume of trapped air was observed in $86 \%$ patients with congenital diaphragmatic hernia, $50 \%$ with meconium aspiration syndrome and $58 \%$ with other diagnoses. After bronchodilation, mean SDS FEV 1 and forced vital capacity were negatively influenced by duration of ventilation (both $p<0.001)$ and duration of ECMO $(p=0.003$ and $p=0.02$, respectively).

Long-term pulmonary sequelae after neonatal ECMO-treatment mainly occur in congenital diaphragmatic hernia patients and tend to deteriorate over time.

KEYWORDS: Chronic lung disease, congenital diaphragmatic hernia, follow-up, longitudinal changes in lung function, long-term sequelae of neonatal lung disease, meconium aspiration syndrome

xtracorporeal membrane oxygenation (ECMO) is a cardiopulmonary bypass technique providing life support when conventional treatment for severe respiratory failure is not enough. Underlying diagnoses include meconium aspiration syndrome (MAS), congenital diaphragmatic hernia $(\mathrm{CDH})$, persistent pulmonary hypertension of the newborn (PPHN), sepsis and pneumonia. Ventilator settings are low during ECMO so the lungs can rest. Lung healing is promoted by reducing barotrauma and hyperoxia [1]. The collaborative UK ECMO trial showed improved survival of term infants with severe respiratory failure who were treated with ECMO [2-5]. The long-term pulmonary sequelae of neonatal ECMO have hardly been studied. Crosssectional studies during or shortly after ECMO all reported reduced lung function, perhaps due to severity of the underlying respiratory disease [6-10].
We report a study in which we longitudinally evaluated residual lung function in neonatal ECMO-treated children now between 5 and 12 yrs of age, distinguished by underlying diagnosis. Furthermore, we related perinatal characteristics to lung function.

\section{METHODS}

\section{Participants}

A prospective longitudinal follow-up study was conducted in children who received veno-arterial ECMO support within the first week of life between February 1991 and August 2004 at the intensive care unit of the Erasmus MC - Sophia Children's Hospital, Rotterdam, the Netherlands. The cohort was supplemented with five children who received veno-arterial ECMO in two other ECMO centres (Nijmegen, the Netherlands, $n=4$; and Leuven, Belgium, $n=1$ ). Inclusion criteria

\section{AFFILIATIONS}

*Intensive Care and Dept of Pediatric Surgery, Erasmus MC - Sophia Children's Hospital, and

\# Dept of Biostatistics, Erasmus MC - Sophia Children's Hospital, and 'Dept of Pediatrics - Respiratory Medicine and Allergology, Erasmus MC - Sophia Children's Hospital, Rotterdam, The Netherlands. +Both authors contributed equally.

CORRESPONDENCE

M. Spoel

Erasmus MC - Sophia Children's Hospital Intensive Care and Dept of Pediatric Surgery

Room Sk 3142

PO Box 2060

3000 CB Rotterdam

The Netherlands

E-mail: m.spoel@erasmusmc.nl

Received:

Nov 012011

Accepted after revision:

March 132012

First published online:

April 102012

European Respiratory Journa Print ISSN 0903-1936 Online ISSN 1399-3003 
and treatment protocols in those centres were the same as ours. ECMO was initiated in case of reversible severe respiratory failure and an estimated mortality risk of higher than $80 \%$ using the entry criteria of STOLAR et al. [11]. We have previously reported our entry criteria and exclusion criteria and these did not change during the study period [10]. The study was embedded in a structured prospective post-ECMO follow-up programme initiated in 2001 that provides for regular assessments of lung function, growth and developmental parameters until 18 yrs of age [12]. Based on the national consensus on neonatal follow-up and the Dutch Ministry of Health's requirement to provide relevant data, the assessment protocol is the standard of care in the Netherlands following ECMO. As a consequence, institutional review board approval was waived. The parents received information about the study and gave written informed consent for analysis of data collected during routine care. Background data were obtained from the charts, including diagnosis, gestational age, birth weight, age at onset of ECMO, duration of ECMO support, duration of mechanical ventilation, highest mean airway pressure (MAP) and highest oxygenation index prior to ECMO, total duration of mechanical ventilation (including ECMO), and duration of oxygen dependency.

Following JOBE and BANCALARI [13], we defined chronic lung disease (CLD) as oxygen dependency at day 28 and classified it as mild, moderate or severe, based on the amount of oxygen needed at day 56 or at discharge, whichever time point was reached first.

\section{Lung function}

Pulmonary function tests were performed at 5, 8, and 12 yrs if children were in a clinically stable condition.

We obtained flow-volume curves; forced expiratory volume in $1 \mathrm{~s}$ (FEV1), forced vital capacity (FVC), and maximum midexpiratory phase (FEF25-75) were determined from the best of three reproducible manoeuvres. At 8 and 12 yrs, we also determined total lung volume, functional residual capacity and residual volume by helium dilution spirometry (TLCspiro, FRCspiro and RV spiro, respectively), and by body plethysmography (TLCpleth, FRCpleth and RVpleth), and carbon monoxide diffusion capacity $(\mathrm{DL}, \mathrm{CO})$, corrected for alveolar volume (KCO) using a single breath method (all equipment: Jaeger Masterlab, Viasys, Hoechberg, Germany). Equipment and procedures were all in accordance with European Respiratory Society criteria [14].

\section{Respiratory morbidity}

At 5, 8 and 12 yrs, a medical history was taken, including information regarding the presence of atopic and respiratory symptoms, and prescription of prophylactic antibiotics, bronchodilators and inhaled corticosteroids medication for pulmonary disease.

\section{Analysis}

The oxygenation index was calculated as: $\left(\left(\mathrm{MAP} \times \mathrm{FI}, \mathrm{O}_{2}\right) / \mathrm{Pa}_{\mathrm{a}} \mathrm{O}_{2}\right)$ $\times 100$, where $\mathrm{FI}_{1} \mathrm{O}_{2}$ is the inspiratory oxygen fraction and $\mathrm{Pa}, \mathrm{O}_{2}$ is the arterial oxygen tension [11]. FEV1, FVC, FEV1/FVC and FEF25-75 were expressed as standard deviation score (SDS) calculated from the reference values of STANOJEVIC et al. [15]. Volume of trapped air is considered significant when the ratio of plethysmographic to spirometric FRC is larger than 1.10 [16].
Post-bronchodilator changes of FEV1 were calculated as a simple percentage of the pre-bronchodilator value: $100 \times$ (post-pre)/ post. A $>11 \%$ bronchodilator change in FEV1 was considered significant, reflecting reversible airflow obstruction [17].

The null hypothesis that the SDS of lung function parameters did not differ from those of the reference population $(S D S=0)$ was tested with the one-sample, unpaired t-test.

Mixed-model ANOVA, which allows for missing data, was applied for the longitudinal evaluation of the spirometric SDS at 5, 8 and 12 yrs and RV/TLC ratios, and the ratio of FRCpleth to FRCspiro and SDS of DL,CO at 8 and 12 yrs [18].

Values for the two largest diagnostic subgroups $(\mathrm{CDH}$ and MAS) were analysed separately. The other subgroups were small and we grouped these as "other diagnoses". Correlation coefficients between lung function parameters and gestational age, birth weight and duration of ventilation, supplemental oxygen and ECMO were established with Spearman's correlation test.

Possible associations between lung function parameters and CLD were explored by univariate analyses. All results are expressed as mean \pm SD or median. $p$-values $<0.05$ were considered significant. Statistical analysis was performed using SPSS 17.0.

\section{RESULTS}

238 children received ECMO support within the first week of life between February 1991 and August 2004 in the Erasmus MC - Sophia Children's Hospital; 166 survived (70\%) (fig. 1). 20 children could not perform reproducible lung function tests. Five children could not be traced, four were followed in another ECMO centre, parents of 15 children gave no consent, and six children had not been tested for logistical reasons. Hence, the study population included $121 \mathrm{ECMO}$ survivors (including the aforementioned five from other centres) who altogether performed 191 pulmonary function tests between February 2002 and March 2011 in Erasmus MC Rotterdam. 70 children (58\%) had been diagnosed with MAS; 20 (17\%) with CDH. Smaller subgroups received ECMO for: PPHN $(n=17)$, sepsis $(n=8)$, pneumonia $(n=4)$ and cardiorespiratory failure $(n=2)$. The characteristics at birth are shown in table 1 . The tested children did not differ from the non-tested children in gestational age, birth weight, underlying diagnosis, highest MAP, highest oxygenation index, age at onset of ECMO or duration of ECMO (data not shown). Atopic symptoms were reported in seven $(9.7 \%)$, nine $(11.7 \%)$ and nine $(21.4 \%)$ children at 5,8 and 12 yrs, respectively. One child at age 5 yrs $(1.4 \%)$ and one child at age $8 \mathrm{yrs}(1.3 \%)$ took antibiotic prophylaxis to prevent recurrent airway infections. At 5 yrs, 10 children (13.9\%) used bronchodilators (four with additional inhaled steroids). At 8 yrs, eight children (10.4\%) used bronchodilators (five with additional inhaled steroids). At 12 yrs, four children (9.8\%) used bronchodilators (two with additional inhaled steroids).

\section{Lung function}

Spirometry

The results of spirometry after bronchodilation are shown in table 2. Significant differences from the norm $(S D S=0)$ are indicated in the table. Significant reversibility of FEV1 was observed in 34 measurements (18\%). The median (interquartile range) change in FEV1 after bronchodilation was 5\% (1-10\%). 


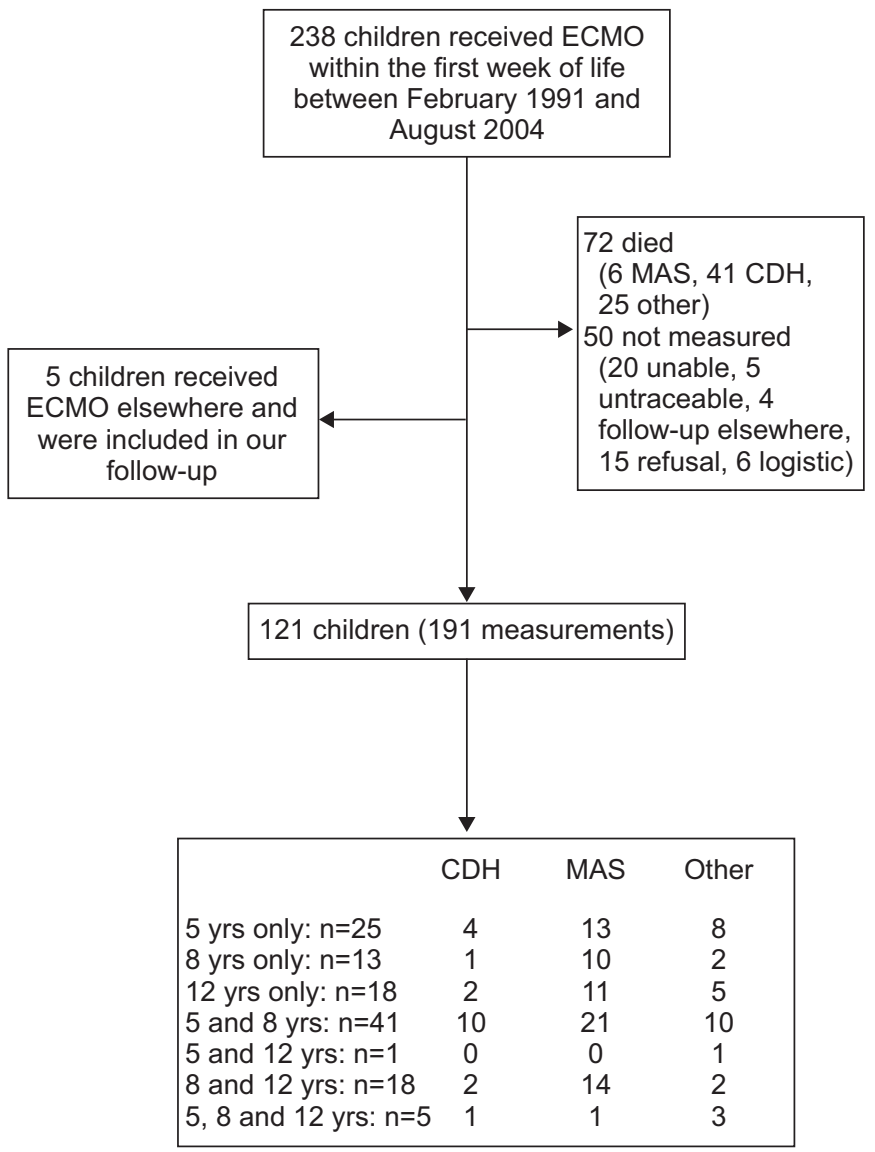

FIGURE 1. Flowchart: children included in the follow-up programme. ECMO: extracorporeal membrane oxygenation; MAS: meconium aspiration syndrome; $\mathrm{CDH}$ : congenital diaphragmatic hernia.
Mean SDS FEV1 before and after bronchodilation significantly changed over time: at 5 yrs it was significantly higher than at 8 yrs ( $p=0.039$ before and $p=0.001$ after bronchodilation) and at 12 yrs ( $p=0.003$ before and $p=0.001$ after bronchodilation). It did not significantly change between 8 and 12 yrs.

Mean SDS FVC before and after bronchodilation did not change significantly over time ( $\mathrm{p}$-values not shown).

Mean SDS FEV1/FVC at 5 yrs was higher than at 8 and 12 yrs, both before and after bronchodilation (all p-values <0.001). After bronchodilation, mean SDS FEV1/FVC did not change significantly between 8 and 12 yrs; before bronchodilation it was higher at 8 yrs $(\mathrm{p}=0.033)$.

Mean SDS FEF25-75 after bronchodilation was higher at 5 yrs than at 8 yrs $(p=0.02)$; it did not change significantly before bronchodilation or from 8 to 12 yrs before and after bronchodilation.

Figures 2 and 3 show the SDS FEV1 at 5, 8 and 12 yrs for the three subgroups and the whole group, before and after bronchodilation. Initial diagnosis was a significant determinant in the mixed model: all spirometric parameters before bronchodilation in the $\mathrm{CDH}$ group were significantly lower than those in the other two subgroups. The parameters did not significantly differ between the MAS group and the other diagnoses group (data not shown). Individual measurements of FEV1, before and after bronchodilation, in $\mathrm{CDH}$ patients are shown in figures 4 and 5 .

We analysed spirometric values in children with repeated measurements separately. After bronchodilation, SDS FEV1 was significantly higher at 5 yrs compared with 8 and $12 \mathrm{yrs}$ $(p=0.002$ and $p=0.005$, respectively). FEV1/FVC was significantly higher at 5 yrs compared with 8 and 12 yrs (both $\mathrm{p}<0.001)$. FEF25-75 was significantly higher at 5 yrs compared

TABLE 1 Clinical perinatal characteristics of neonatal extracorporeal membrane oxygenation (ECMO)-treated patients

\begin{tabular}{|c|c|c|c|c|}
\hline & All participants & MAS & CDH & Other \\
\hline Subjects n (males) & $121(65)$ & $70(33)$ & $20(15)$ & $31(17)$ \\
\hline Birth weight $\mathbf{g}$ & $3380(2160-4980)$ & $3400(2300-4980)$ & $3500(2160-3810)$ & $3280(2375-4880)$ \\
\hline Age at onset ECMO $h$ & $25(5-168)$ & $23(6-73)$ & $16(5-168)$ & $40(15-152)$ \\
\hline Duration of ECMO $h$ & $132(24-369)$ & $126(24-345)$ & $192(68-369)$ & $122(53-288)$ \\
\hline Highest OI prior to ECMO & $43(15-143)$ & $44(27-143)$ & $44(15-130)$ & $40(21-106)$ \\
\hline NO treated newborns & $80(66)$ & $42(60)$ & $13(65)$ & $25(81)$ \\
\hline CLD & 22 (18.2) & $12(17.2)$ & $10(50.0)$ & \\
\hline Mild CLD & $11(9.1)$ & $10(14.3)$ & $1(5.0)$ & \\
\hline Moderate CLD & $1(0.8)$ & & $1(5.0)$ & \\
\hline
\end{tabular}

Data are expressed as median (range) or $\mathrm{n}(\%)$, unless otherwise indicated. Shown are the total group and the subgroups of infants. MAS: meconium aspiration syndrome; $\mathrm{CDH}$ : congenital diaphragmatic hernia; MAP: mean airway pressure; OI: oxygenation index; NO: nitric oxide therapy; CLD: chronic lung disease, classified according to JOBE and BANCALARI [13]. 


\begin{tabular}{|c|c|c|c|}
\hline \multirow[t]{2}{*}{ TABLE 2} & \multicolumn{3}{|c|}{$\begin{array}{l}\text { Longitudinal results of spirometry after neonatal } \\
\text { extracorporeal membrane oxygenation, after } \\
\text { bronchodilation }\end{array}$} \\
\hline & $5 \mathrm{yrs}$ & 8 yrs & $12 \mathrm{yrs}$ \\
\hline $\begin{array}{l}\text { Subjects } n \\
\text { SDS FEV } 1\end{array}$ & 72 & 77 & 42 \\
\hline All participants & $0.07 \pm 0.14$ & $-0.40 \pm 0.15^{\star}$ & $-0.52 \pm 0.16^{*}$ \\
\hline MAS & $0.49 \pm 0.17^{\star *}$ & $0.12 \pm 0.14$ & $0.01 \pm 0.23$ \\
\hline $\mathrm{CDH}$ & $-0.71 \pm 0.40$ & $-2.27 \pm 0.36^{\#}$ & $-2.73 \pm 0.61^{*}$ \\
\hline Other & $0.01 \pm 0.23$ & $0.08 \pm 0.31$ & $-0.49 \pm 0.25$ \\
\hline \multicolumn{4}{|l|}{ SDS FVC } \\
\hline All participants & $-0.08 \pm 0.15$ & $-0.22 \pm 0.13$ & $-0.29 \pm 0.16$ \\
\hline MAS & $0.40 \pm 0.18^{*}$ & $0.01 \pm 0.15$ & $0.12 \pm 0.27$ \\
\hline $\mathrm{CDH}$ & $-0.69 \pm 0.43$ & $-1.48 \pm 0.35^{\#}$ & $-1.28 \pm 0.98$ \\
\hline Other & $-0.01 \pm 0.29$ & $0.19 \pm 0.25$ & $-0.17 \pm 0.33$ \\
\hline \multicolumn{4}{|l|}{ SDS FEV $1 / F V C$} \\
\hline All participants & $0.32 \pm 0.15$ & $-0.53 \pm 0.12^{\#}$ & $-0.63 \pm 0.15^{\#}$ \\
\hline MAS & $0.22 \pm 0.19$ & $-0.19 \pm 0.11$ & $-0.32 \pm 0.19$ \\
\hline $\mathrm{CDH}$ & $0.11 \pm 0.35$ & $-1.47 \pm 0.39^{* *}$ & $-2.16 \pm 0.30^{* *}$ \\
\hline Other & $0.21 \pm 0.35$ & $-0.37 \pm 0.28$ & $-0.61 \pm 0.34$ \\
\hline \multicolumn{4}{|l|}{ SDS FEF25-75 } \\
\hline All participants & $-0.56 \pm 0.19^{\star \star}$ & $-1.02 \pm 0.18^{\#}$ & $-0.97 \pm 0.20^{\#}$ \\
\hline MAS & $-0.12 \pm 0.25$ & $-0.34 \pm 0.14^{*}$ & $-0.67 \pm 0.21^{* *}$ \\
\hline $\mathrm{CDH}$ & $-1.76 \pm 0.42^{\star *}$ & $-3.07 \pm 0.46^{\#}$ & $-3.28 \pm 0.54^{\star *}$ \\
\hline Other & $-0.82 \pm 0.38$ & $-0.71 \pm 0.42$ & $-0.93 \pm 0.30^{*}$ \\
\hline
\end{tabular}

Mean \pm SE standard deviation scores (SDS) are shown for FEV1 (forced expiratory volume in $1 \mathrm{~s}$ ), FVC (forced vital capacity), FEV1/FVC, and FEF25-75 (maximum mid-expiratory phase). Number of patients studied in each group: meconium aspiration syndrome (MAS): 35,46 and 26 at 5, 8 and $12 \mathrm{yrs}$; congenital diaphragmatic hernia (CDH): 15,14 and 5 at 5,8 and 12 yrs; others: 22, 17 and 11 at 5,8 and 12 yrs. SDS significantly below normal (SDS=0; onesample t-test), ${ }^{*}: p<0.05 ;{ }^{* *}: p<0.01 ;{ }^{\#}: p \leqslant 0.001$.

with 8 yrs $(p=0.028)$; there was no significant difference compared with 12 yrs. This is similar to the analysis of the whole group, including children with only one measurement. For all lung function parameters, differences between the different subgroups were independent of age.

\section{Helium dilution spirometry, body plethysmography and diffusion capacity at $\mathbf{8}$ and 12 yrs}

The mean \pm SD RV/TLCspiro at 8 yrs was $22.1 \pm 8.3 \%$; at 12 yrs it was $21.1 \pm 7.0 \%$. The mean \pm SD RV/TLCpleth at 8 yrs was $29.4 \pm 8.2 \%$; at 12 yrs it was $26.5 \pm 6.8 \%$ (table 3 ).

The mean \pm SD FRCpleth/spiro was $1.22 \pm 0.22$ at 8 yrs and $1.09 \pm 0.11$ at $12 \mathrm{yrs}$. A significant volume trapped air (defined as FRCpleth/spiro >1.10) [16] was observed in 32 (64\%) and 13 (46\%) children at 8 and 12 yrs, respectively. This concerned 12 out of 14 measurements in the CDH group (86\%), 26 out of 52 in the MAS group (50\%), and seven out of 12 in the other diagnoses group (58\%).

Total diffusion capacity did not differ from the normal population at 8 and 12 yrs ( $\mathrm{p}=0.286$ and $\mathrm{p}=0.392$, respectively). However, after correction for alveolar volume, the diffusion

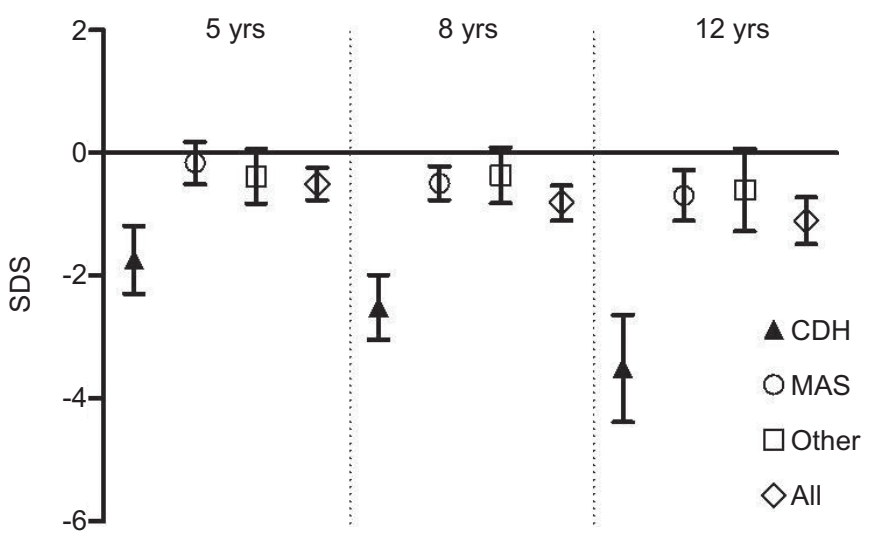

FIGURE 2. Standard deviation scores (SDS) forced expiratory volume in $1 \mathrm{~s}$ (mean and 95\% confidence interval) before bronchodilation at 5, 8 and $12 \mathrm{yrs}$ for the different subgroups. Triangles represent congenital diaphragmatic hernia $(\mathrm{CDH})$ patients ( $n=14,13$ and 5 at 5, 8 and $12 \mathrm{yrs}$, respectively); circles represent children with meconium aspiration syndrome (MAS) $(n=34,46$, and 24 at 5,8 and $12 \mathrm{yrs,}$ respectively), squares represent children who underwent neonatal extracorporeal membrane oxygenation for other diagnoses $(n=22,16$ and 9 at 5,8 and 12 yrs, respectively). A summary of all cases is shown represented by the diamond symbols.

capacity was significantly below the norm at the age of 8 $(\mathrm{p}<0.001)$ but not at 12 yrs $(\mathrm{p}=0.172)$.

\section{Other determinants of lung function parameters}

Beside the effects of age and initial diagnosis we evaluated the influence of other determinants on spirometric parameters. Before bronchodilation, mean SDS FEV1 and SDS FVC were negatively influenced by duration of ventilation (both parameters $p<0.001)$, duration of ECMO support $(p \leqslant 0.001$ for both parameters), and the presence of CLD (both $p \leqslant 0.001$ ). Both parameters were positively correlated to birth weight (both

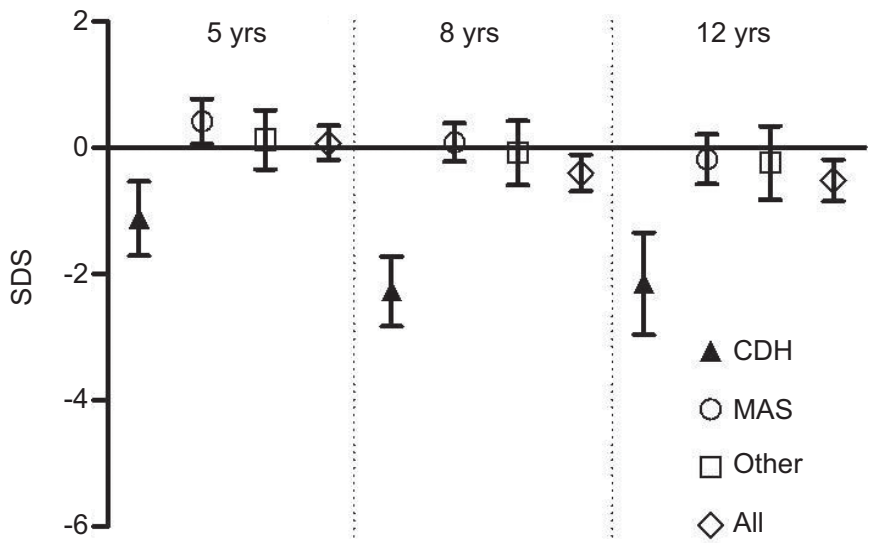

FIGURE 3. Standard deviation scores (SDS) of forced expiratory volume in $1 \mathrm{~s}$ (mean and 95\% confidence interval) after bronchodilation at 5, 8 and 12 yrs for the different subgroups. Triangles represent congenital diaphragmatic hernia $(\mathrm{CDH})$ patients ( $n=10,14$ and 5 at 5,8 and $12 \mathrm{yrs}$, respectively); circles represent children with meconium aspiration syndrome (MAS) ( $n=26,46$ and 23 at 5,8 and $12 \mathrm{yrs}$, respectively), squares represent children who underwent neonatal extracorporeal membrane oxygenation for other diagnoses $(n=16,15$ and 11 at 5,8 and $12 \mathrm{yrs}$, respectively). A summary of all cases is shown represented by the diamond symbols. 


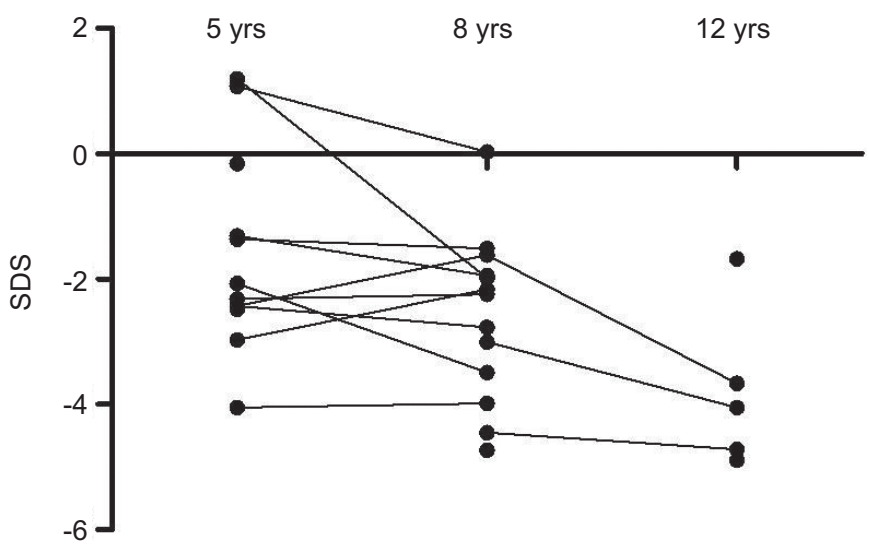

FIGURE 4. Change of standard deviation scores (SDS) of forced expiratory volume in $1 \mathrm{~s}$ before bronchodilation in congenital diaphragmatic hernia patients from 5 to 12 yrs. Each dot represents a measurement of an individual patient. $n=14$ at $5 \mathrm{yrs}, \mathrm{n}=13$ at $8 \mathrm{yrs}$ and $\mathrm{n}=5$ at $12 \mathrm{yrs}$.

$\mathrm{p} \leqslant 0.001)$; SDS FEV1 only was positively correlated to gestational age $(\mathrm{p}=0.001)$.

After bronchodilation, mean SDS FEV1, SDS FVC and SDS FEF25-75 were negatively influenced by duration of ventilation (all parameters $\mathrm{p}<0.001$ ), duration of ECMO support (all $p<0.03$ ), and the presence of CLD (all $p \leqslant 0.001$ ). Mean SDS FEV1, SDS FVC, SDS FEV1/FVC and SDS FEF25-75 were positively influenced by birth weight (all $\mathrm{p}<0.05$ ); mean SDS FEV1 and SDS FEF25-75 were also positively influenced by gestational age (both $\mathrm{p}<0.01$ ). In univariate analysis, doubling of the logarithm ventilation time resulted in a mean decrease of -0.415 SDS FEV1 at 5 yrs, -0.782 SDS FEV1 at 8 yrs, and -1.35 SDS FEV1 at 12 yrs (all $\mathrm{p} \leqslant 0.001)$.

$\mathrm{RV} / \mathrm{TLC}$ leth and FRCpleth/spiro were positively correlated to duration of ventilation $(\mathrm{p}<0.001)$, duration of ECMO support $(p<0.02)$, and birth weight $(p<0.04)$. Highest MAP and oxygenation index before ECMO did not correlate with any of the lung function parameters.

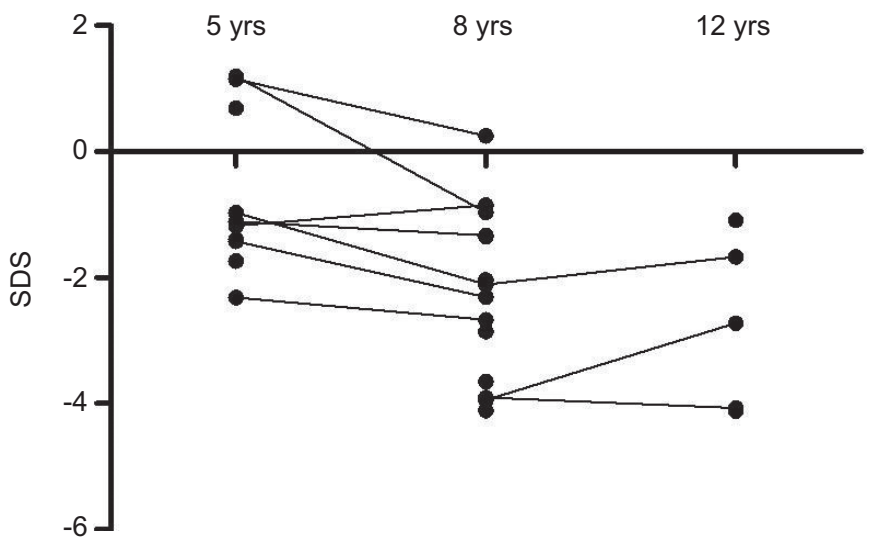

FIGURE 5. Change of standard deviation scores (SDS) of forced expiratory volume in $1 \mathrm{~s}$ after bronchodilation in congenital diaphragmatic hernia patients from 5 to 12 yrs. Each dot represents a measurement of an individual patient. $n=10$ at $5 \mathrm{yrs}, \mathrm{n}=14$ at $8 \mathrm{yrs}$ and $\mathrm{n}=5$ at $12 \mathrm{yrs}$

\begin{tabular}{lcccc} 
TABLE 3 & $\begin{array}{c}\text { Static lung volumes and diffusion capacity at } 8 \\
\text { and } 12 \text { yrs }\end{array}$ \\
& $\mathbf{8}$ yrs & Subjects $\mathbf{n}$ & $\mathbf{1 2}$ yrs & Subjects $\mathbf{~}$ \\
\hline & & & & \\
$\mathbf{R V} / \mathbf{T L C}$ spiro \% & $22.1 \pm 8.3$ & 56 & $21.1 \pm 7.0$ & 30 \\
$\mathbf{R V} / \mathbf{T L C}$ pleth \% & $29.4 \pm 8.2$ & 40 & $26.5 \pm 6.8$ & 25 \\
$\mathbf{F R C}$ pleth/spiro & $1.22 \pm 0.22$ & 50 & $1.09 \pm 0.11$ & 28 \\
$\mathbf{V} \mathbf{A}$ & $32(64)$ & & $13(46)$ & \\
$\mathbf{D L}, \mathbf{C O}_{\mathbf{c}}$ SDS & $0.32 \pm 1.6$ & 29 & $-0.28 \pm 1.5$ & 22 \\
$\mathbf{K C O}_{\mathbf{c}}$ SDS & $-0.95 \pm 1.1^{* * *}$ & 22 & $-0.41 \pm 1.2$ & 18 \\
\hline
\end{tabular}

Data are presented as mean \pm SD or $n(\%)$, unless otherwise stated. RV: residual volume; TLC: total lung capacity; spiro: determined by helium dilution spirometry; pleth: determined by body plethysmography; FRC: functional residual capacity; VTA: volume trapped air; $\mathrm{DL}, \mathrm{CO}_{\mathrm{c}}$ : diffusing capacity of the lung for carbon monoxide, corrected for haemoglobin; $\mathrm{KCO}_{\mathrm{C}}$ : diffusion capacity corrected for alveolar volume and haemoglobin. For VTA, the number (\%) of patients with significant VTA (defined as FRCpleth/spiro >1.10) is shown. ***: $p<0.001$, significantly below normal $(S D S=0)$ at $8 \mathrm{yrs}$.

\section{DISCUSSION}

Residual lung function of the studied 121 children, in terms of mean SDS FEV1 and SDS FEF25-75 before bronchodilation, had significantly decreased between 5 and 12 yrs of age. CDH was associated with significantly lower spirometric values and higher frequency of a significant volume of trapped air compared to other diagnoses. Mean SDS FEV1, FVC and FEF25-75 were negatively influenced by duration of ventilation, and the presence of CLD.

Long-term pulmonary function abnormalities, such as hyperinflation and airway obstruction, are well recognised after neonatal respiratory failure secondary to lung injury from MAS, $\mathrm{CDH}$ and neonatal pneumonia [10, 19-20]. Treatment modalities such as supplemental oxygen and mechanical ventilation contribute to the pathogenesis of CLD [21]. Avoiding continued exposure to high inspired oxygen concentration and barotrauma during the course of ECMO has reduced mortality and encouraged lung healing and recovery. To our knowledge this is the first longitudinal study on the impact of neonatal ECMO on lung function in children between 5 and 12 yrs of age.

In 2000, BEARDSMORE et al. [6] cross-sectionally studied 51 ECMO patients at age $1 \mathrm{yr}$ and found few differences in lung function compared with conventionally ventilated controls. This provided reassurance that in addition to decreased mortality respiratory function following ECMO was no worse. In addition, another study by BEARDSMORE et al. [22] showed that, when children were categorised according to the underlying reason for ECMO, those treated for respiratory distress syndrome and those treated beyond the first 3 weeks for bronchiolitis or pneumonia had poorer pulmonary function 12 months later. However, few CDH patients were included in this studied population of 106 subjects [22]. HoFHUIS et al. [10] found below-average but normal lung volumes and stable forced expiratory flows during the first year of life in 64 infants following ECMO. At 12 months, only the $\mathrm{CDH}$ patients showed signs of hyperinflation with plethysmographic FRC significantly above normal. In an earlier study we found significantly 
impaired expiratory flows and increased FRC levels in 12 ECMO and 31 non-ECMO treated $\mathrm{CDH}$ patients during the first year of life. The ECMO-treated $\mathrm{CDH}$ patients had significantly higher FRC levels, thus reflecting more hyperinflation [20]. In a cross-sectional study in 54 8-yr-old patients after severe neonatal respiratory failure, MAJAESIC et al. [23] found a poorer pulmonary outcome in the subgroup of ECMO treated CDH patients. In 2004, HAMUTCU et al. [19] cross-sectionally studied 50 children after neonatal ECMO treatment. At a mean age of 11 yrs they had significantly lower FEV1 and FEF25-75 and hyperinflation with higher RV compared to healthy matched controls. Single breath diffusion capacity for carbon monoxide was within the normal range. When children with congenital heart disease $(8 \%)$ and $\mathrm{CDH}(12 \%)$ were excluded from analysis, no significant differences in lung function were observed. BOYKIN et al. [7] reported signs of air trapping and mild lower airway obstruction in 17 ECMO-treated MAS patients.

The strength of the present study is the longitudinal aspect, as most other studies were performed cross-sectionally. A potential weakness is the lack of appropriate reference values for longitudinal spirometric measurements and the lack of a healthy control group in our study. Up-to-date reference data are needed to reflect evolving measurement techniques and equipment and changes in population characteristics. In 2008, QUANJER et al. [24] compared five commonly used reference equation sets to serial measurements and found that Stanojevic's equations performed best and are suitable for longitudinal data analysis as they cover a wide age range and account for a gradual transition from childhood into adulthood. In another study they compared 30 spirometry datasets and concluded that the use of local controls to validate reference equations will rarely be practical due to the numbers required and that the use of reference equations derived from large or collected datasets is recommended [25]. Therefore we computed SDS of spirometric values using those equations, which distinguish between the effects of disease and those of growth and development [24]. As treatment protocols improve over time, results from earlier studies may not just be applicable to patients treated with ECMO today. This is, albeit to a lower extent, also a limitation of our study. Not all patients were tested at all three time points, which created an unfavourable but unavoidable heterogeneity in the age distribution. Our ventilation strategies and ECMO treatment protocols have indeed been adjusted over the past decade, resulting in better survival in $\mathrm{CDH}$ patients [26]. Recent protocols based on meta-analysis of retrospective studies provide guidelines for the use of ECMO in CDH patients [27]. These could well lead to better long-term pulmonary outcome and lung function.

In our study, the SDS for all spirometric parameters in the CDH group were significantly lower than those in patients with MAS or other diagnoses. This finding is in line with findings from other studies. While infants with MAS have normal lung development, $\mathrm{CDH}$ is associated with lung hypoplasia and PPHN. The severity of lung hypoplasia and PPHN in $\mathrm{CDH}$ patients covers a wide range. ECMO-treated $\mathrm{CDH}$ patients are regarded as the most severe cases [20, 26]. Their improved survival might be counterbalanced by increased morbidity. Maldevelopment of the alveoli and pulmonary vessels with disturbed lung growth may be responsible for the deterioration of lung function. Also, prolonged ventilatory support and supplemental oxygen after ECMO treatment may result in CLD. Recurrent respiratory tract infections can further compromise lung function over time.

Our group has recently reported a significant decline of exercise capacity in a cohort of ECMO survivors which overlaps with the population described in the present study [28]. Interestingly, this decline was irrespective of the underlying diagnosis and we were unable to show an association between maximal exercise capacity and SDS of FEV1 and FEV1/FVC. For all patients, we advocate an active lifestyle and healthy eating pattern, as sports participation interacts positively and BMI negatively with exercise capacity [29].

Adequate treatment of recurrent respiratory tract infections, close monitoring and treatment of asthma could perhaps halt deterioration of exercise capacity and lung function, especially in $\mathrm{CDH}$ patients. The use of prophylactic bronchodilators, e.g. during exercise, should not be advised routinely.

From our results it can be concluded that lung function is compromised after neonatal ECMO treatment and seems to deteriorate over time in $\mathrm{CDH}$ patients only. Airway patency in patients with all other diagnoses was within the normal range and remained stable over time. Therefore we assume that the underlying condition and not ECMO itself is responsible for the compromised lung function post-ECMO. ECMO may even reduce the harmful effects of high pressure ventilation and high doses of oxygen. Although the severity of pulmonary hypertension diminishes during ECMO and later in $\mathrm{CDH}$ patients, sequelae of abnormal lung development will still be present as reflected by compromised lung function in this patient group.

We recommend prolonged follow-up, especially of patients with $\mathrm{CDH}$, to further elucidate the increased respiratory morbidity that occurs with better survival and changing treatment protocols.

\section{SUPPORT STATEMENT}

The Swart-van Essen foundation financially supported this study.

\section{STATEMENT OF INTEREST}

None declared.

\section{ACKNOWLEDGEMENTS}

The authors thank the staff of the lung function lab from the Erasmus MC, Rotterdam, the Netherlands for their hospitality and cooperation. K. Hagoort (Erasmus MC - Sophia Children's Hospital, Rotterdam) provided editorial advice.

\section{REFERENCES}

1 Mugford M, Elbourne D, Field D. Extracorporeal membrane oxygenation for severe respiratory failure in newborn infants. Cochrane Database Syst Rev 2008: CD001340.

2 UK collaborative randomised trial of neonatal extracorporeal membrane oxygenation. UK Collaborative ECMO Trail Group. Lancet 1996; 348: 75-82.

3 The collaborative UK ECMO (Extracorporeal Membrane Oxygenation) trial: follow-up to 1 year of age. Pediatrics 1998; 101: E1.

4 Bennett CC, Johnson A, Field DJ, et al. UK collaborative randomised trial of neonatal extracorporeal membrane oxygenation: follow-up to age 4 years. Lancet 2001; 357: 1094-1096.

5 McNally H, Bennett CC, Elbourne D, et al. United Kingdom collaborative randomized trial of neonatal extracorporeal membrane 
oxygenation: follow-up to age 7 years. Pediatrics 2006; 117: e845-e854.

6 Beardsmore C, Dundas I, Poole K, et al. Respiratory function in survivors of the United Kingdom Extracorporeal Membrane Oxygenation Trial. Am J Respir Crit Care Med 2000; 161: 1129-1135.

7 Boykin AR, Quivers ES, Wagenhoffer KL, et al. Cardiopulmonary outcome of neonatal extracorporeal membrane oxygenation at ages 10-15 years. Crit Care Med 2003; 31: 2380-2384.

8 Garg M, Kurzner SI, Bautista DB, et al. Pulmonary sequelae at six months following extracorporeal membrane oxygenation. Chest 1992; 101: 1086-1090.

9 Greenspan JS, Antunes MJ, Holt WJ, et al. Pulmonary sequelae in infants treated with extracorporeal membrane oxygenation. Pediatr Pulmonol 1997; 23: 31-38.

10 Hofhuis W, Hanekamp MN, Ijsselstijn $\mathrm{H}$, et al. Prospective longitudinal evaluation of lung function during the first year of life after extracorporeal membrane oxygenation. Pediatr Crit Care Med 2011; 12: 159-164.

11 Stolar CJ, Snedecor SM, Bartlett RH. Extracorporeal membrane oxygenation and neonatal respiratory failure: experience from the extracorporeal life support organization. J Pediatr Surg 1991; 26: 563-571.

12 Hanekamp MN, Mazer P, van der Cammen-van Zijp MH, et al. Follow-up of newborns treated with extracorporeal membrane oxygenation: a nationwide evaluation at 5 years of age. Crit Care 2006; 10: R127.

13 Jobe AH, Bancalari E. Bronchopulmonary dysplasia. Am J Respir Crit Care Med 2001; 163: 1723-1729.

14 Miller MR, Hankinson J, Brusasco V, et al. Standardisation of spirometry. Eur Respir J 2005; 26: 319-338.

15 Stanojevic S, Wade A, Stocks J, et al. Reference ranges for spirometry across all ages: a new approach. Am J Respir Crit Care Med 2008; 177: 253-260.

16 Eber E ZM. Pediatric pulmonary function testing. Prog Respir Res 2005; 33: 94-102.

17 Casan P, Roca J, Sanchis J. Spirometric response to a bronchodilator. Reference values for healthy children and adolescents. Bull Eur Physiopathol Respir 1983; 19: 567-569.

18 Fitzmaurice G, Laird N, Ware J. Applied Longintudinal Analysis. 1st Edn. Hoboken, John Wiley and Sons, 2004.
19 Hamutcu R, Nield TA, Garg M, et al. Long-term pulmonary sequelae in children who were treated with extracorporeal membrane oxygenation for neonatal respiratory failure. Pediatrics 2004; 114: 1292-1296.

20 Spoel M, van den Hout L, Gischler SJ, et al. Prospective longitudinal evaluation of lung function during the first year of life after repair of congenital diaphragmatic hernia. Pediatr Crit Care Med 2012; 13: e133-e139.

21 Stocks J, Godfrey S. The role of artificial ventilation, oxygen, and CPAP in the pathogenesis of lung damage in neonates: assessment by serial measurements of lung function. Pediatrics 1976; 57: 352-362.

22 Beardsmore CS, Westaway J, Killer $\mathrm{H}$, et al. How does the changing profile of infants who are referred for extracorporeal membrane oxygenation affect their overall respiratory outcome? Pediatrics 2007; 120: e762-e768.

23 Majaesic CM, Jones R, Dinu IA, et al. Clinical correlations and pulmonary function at 8 years of age after severe neonatal respiratory failure. Pediatr Pulmonol 2007; 42: 829-837.

24 Quanjer PH, Borsboom GJ, Kivastik J, et al. Cross-sectional and longitudinal spirometry in children and adolescents: interpretative strategies. Am J Respir Crit Care Med 2008; 178: 1262-1270.

25 Quanjer PH, Stocks J, Cole TJ, et al. Influence of secular trends and sample size on reference equations for lung function tests. Eur Respir J 2011; 37: 658-664.

26 van den Hout L, Schaible T, Cohen-Overbeek TE, et al. Actual outcome in infants with congenital diaphragmatic hernia: the role of a standardized postnatal treatment protocol. Fetal Diagn Ther 2011; 29: 55-63.

27 Reiss I, Schaible T, van den Hout L, et al. Standardized postnatal management of infants with congenital diaphragmatic hernia in Europe: the CDH EURO Consortium consensus. Neonatology 2010; 98: 354-364.

28 van der Cammen-van Zijp MH, Gischler SJ, Hop WC, et al. Deterioration of exercise capacity after neonatal extracorporeal membrane oxygenation. Eur Respir J 2011; 38: 1098-1104.

29 van der Cammen-van Zijp $\mathrm{MH}$, van den Berg-Emons RJ, Willemsen SP, et al. Exercise capacity in Dutch children: new reference values for the Bruce treadmill protocol. Scand J Med Sci Sports 2010; 20: e130-e136. 